\title{
Analysis of Dead Zone of RFID System
}

\author{
Mitsuo Taguchi ${ }^{(1)}$, and Hiroyuki Mizuno ${ }^{*(2)}$ \\ (1) Dept. of Electrical \& Electronic Eng., Nagasaki University \\ (2) Graduate School of Science \& Technology, Nagasaki University \\ 1-14 Bunkyo-machi, Nagasaki 852-8521, Japan \\ E-mail:mtaguchi@net.nagasaki-u.ac.jp
}

\section{Introduction}

Recently, the RFID (Radio Frequency Identification) systems have become very popular in many service industries, purchasing and manufacturing companies. When, however, the transponder without its own voltage supply is located near the conducting plate, the reader may not receive the ID signal from the transponder even it is within the interrogation zone of a reader.

In this paper, the dead zone of RFID system is examined. First, the electric field distribution near the square conducting plate is numerically obtained when the plane wave is incident. The current distribution on the conducting plate in the case of spherical wave incidence is expressed by the integration of induced current due to the plane wave incidence. The scattered electric field is calculated and discussed. The electric field distribution near the conducting plate is measured and the existence of the dead zone is shown.

\section{Numerical Analysis}

1. Plane wave incidence

Fig.1 shows the analytical model. The plane wave with horizontal polarization is incident to the square conducting plate of $60 \mathrm{~cm}$ by $60 \mathrm{~cm}$. The frequency is 2.45 GHz. The incident angle is $0^{\circ} \leq \theta \leq 180^{\circ}, 0^{\circ} \leq \phi \leq 180^{\circ}$. The current distribution on the square conductor is calculated by using the electromagnetic simulator WIPL-D based on the Method of Moment [1]. Then the electric field distribution in xy-plane is calculated by the integration of induced current on the conducting plate.

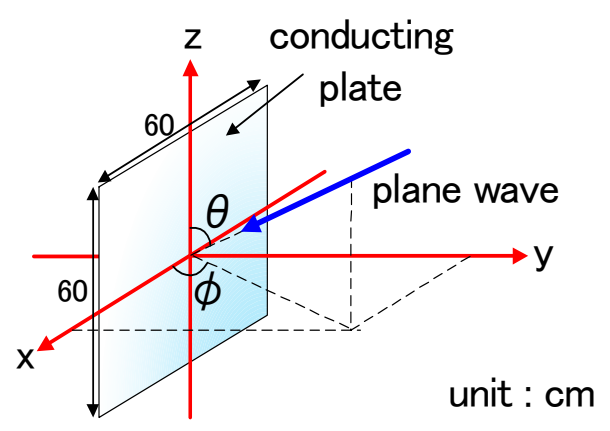

Fig. 1 Analytical model

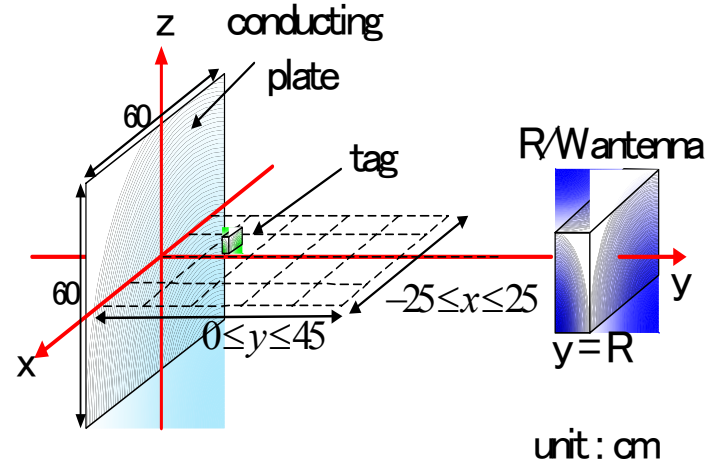

Fig. 2 Experimental model 
2. Spherical wave incidence

Let $\mathbf{J}(\theta, \phi)$ be the current distribution at the point (x, z) on the conducting plate in the case of the plane wave incidence, where $\theta, \phi$ are the incident angles. Then the current distribution $\mathbf{J}_{s p h}$ on the conducting plate in the case of spherical wave incidence is expressed by the plane wave expansion of spherical wave as follows [2].

$$
\begin{aligned}
\mathbf{J}_{s p h}=\left(-\frac{k}{4 \pi}+\frac{1}{8 R}\right) \int_{0}^{\pi} \int_{0}^{\pi} \mathbf{J}(\theta, \phi) \exp ( & \left.j \frac{\pi}{2} \cos \phi \sin \theta\right) \sin \theta d \phi d \theta \\
& -j \frac{k}{4 \pi} \int_{0}^{\pi} \int_{0}^{\pi} \mathbf{J}(\theta, \phi) \sin \theta d \phi d \theta
\end{aligned}
$$

Where the spherical-wave is radiated from the point $\mathrm{y}=\mathrm{R}$ on the $\mathrm{y}$ axial. The scattered electric field from the conductor is calculated by the integration of the current distribution obtained from the equation (1).

\section{Measurement}

Fig. 2 shows the experimental model of RFID system. The 4 by 4 square patch microstrip array antenna is used for the reader antenna. The operating frequency is $2.45 \mathrm{GHz}$. As the passive transponder, the microstrip antenna with finite ground plane is used. The transponder is moved at the interval of $1 \mathrm{~cm}$ in the region of $-25 \mathrm{~cm} \leq x \leq 25 \mathrm{~cm}, 0 \leq y \leq 45 \mathrm{~cm}$ on the xy-plane. The reader reads the ID signal 256 times and records the detection error rate.

\section{Results and Discussion}

Fig. 3 shows the example of calculated electric field distribution in xy-plane in the case of vertical incidence of plane wave. Fig. 4 shows the scattered electric field distribution for the spherical wave incidence from $\mathrm{R}=60 \mathrm{~cm}$ on $\mathrm{y}$-axis. The scattered electric field is calculated from the current $\mathbf{J}_{s p h}$. Fig. 5 shows the calculated phase distribution of scattered electric field at $0 \mathrm{~cm} \leq x \leq 30 \mathrm{~cm}$ along the $\mathrm{x}$ direction and $0 \mathrm{~cm} \leq z \leq 30 \mathrm{~cm}$ along $\mathrm{z}$ direction in the vicinity of conducting

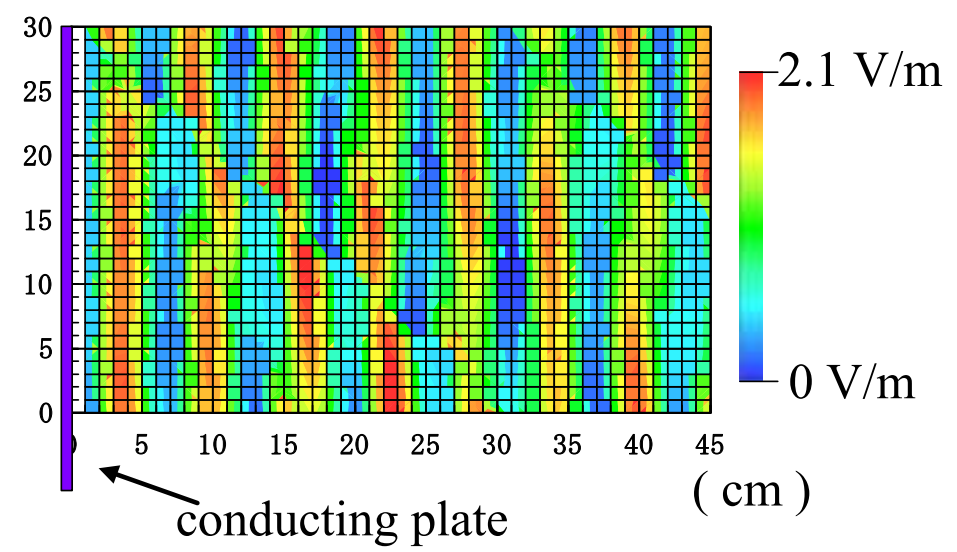

Fig. 3 Electric field distribution in xy-plane for incident angle $\theta=\phi=0^{\circ}$ 
plate. Since the phase delay becomes 180 degrees at the point $x=27.5 \mathrm{~cm}, z=0$ and $x=0, \mathrm{z}=27.5 \mathrm{~cm}$ in the case of spherical wave incidence, the equation (1) of the current is valid. Fig. 6 shows the measured detection error rate for $\mathrm{R}=189 \mathrm{~cm}$. Fig. 7 shows the measured detection error rate for $\mathrm{R}=296 \mathrm{~cm}$. In both figures, the white area indicates the detection error rate greater than 20\%. In Figs. 3, 6 and 7, the dead zones are observed at the interval of $6 \mathrm{~cm}$.

\section{Conclusion}

The dead zone in the RFID system has been numerically and experimentally investigated. The induced current on the conducting plate in the case of spherical wave incidence has been calculated in terms of the current for the plane wave incidence by using the plane wave expansion of spherical wave. The scattered electric field distribution near the finite conducting plate has been obtained from the induced current on the conducting plate. The detection error rate of the RFID system has been measured.

The radiation field from the reader antenna can be expressed in terms of the spherical wave. Therefore the scattered field distribution is straightforwardly calculated from the induced current in the case of plane wave incidence.

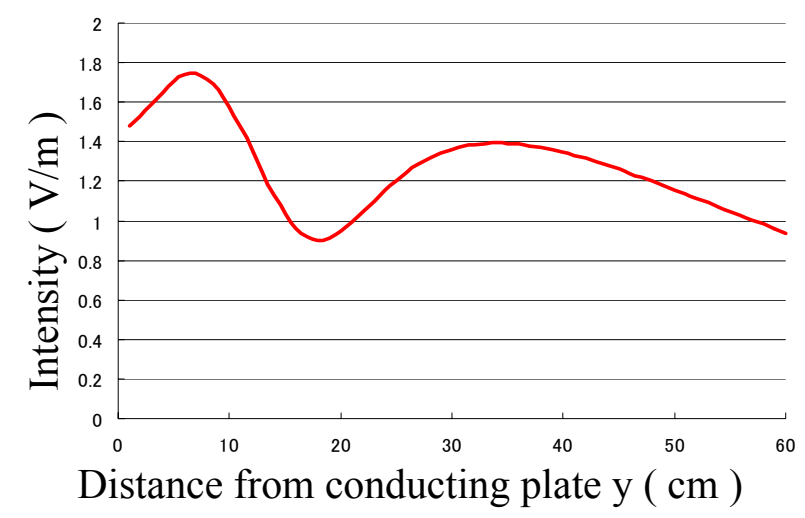

Fig. 4 Scattered electric field

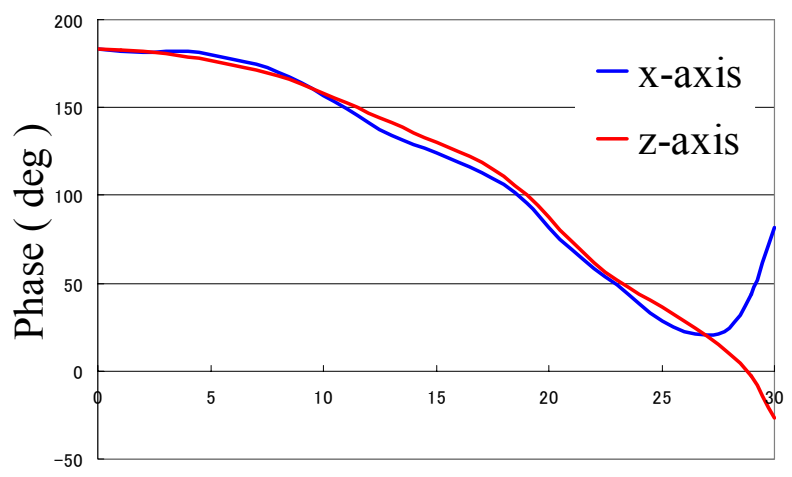

Distance from center in $\mathrm{x}$ or $\mathrm{y}$ direction $(\mathrm{cm})$

Fig. 5 Phase distribution of scattered electric field 


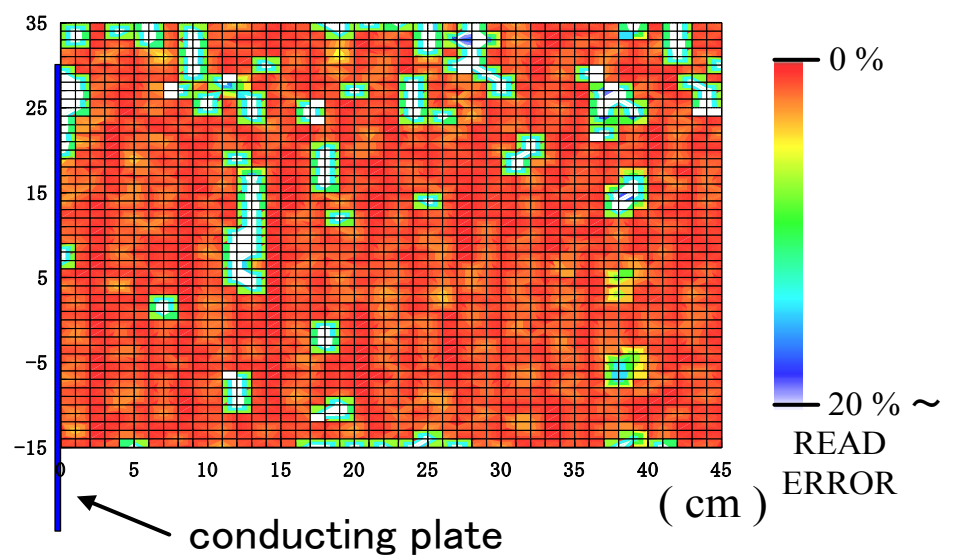

Fig. 6 Detection error rate. $\mathrm{R}=189 \mathrm{~cm}$

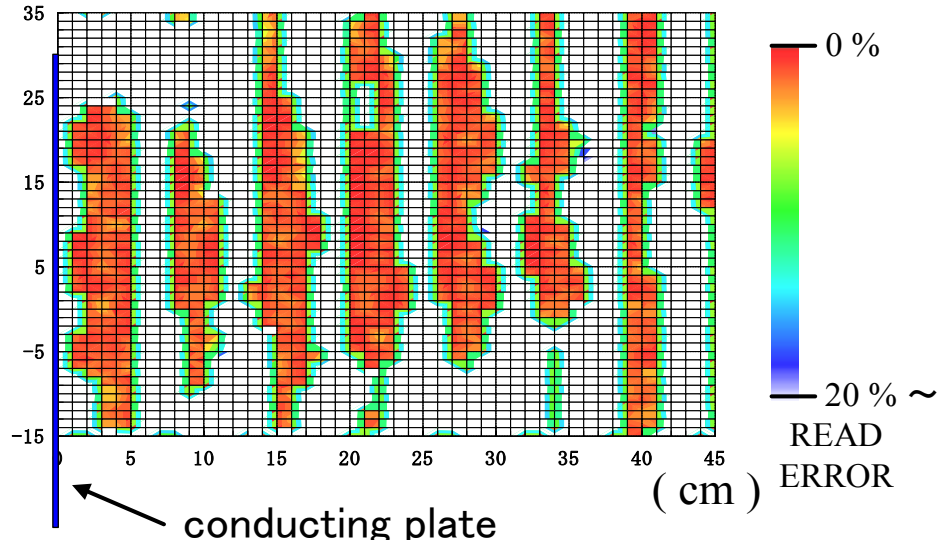

Fig. 7 Detection error rate. $R=296 \mathrm{~cm}$

\section{References:}

[1] http://www.wipl-d.com/products/pro.php

[2] R. Harrington: "Time-harmonic electromagnetic fields", McGraw-Hill, New York, 1961. 